\section{CONTRIBUIÇĀO AO ESTUDO DOS ELEMENTOS VASCULARES, ARTERIAIS E VENOSOS, HILARES, JUSTAHILARES E EXTRAHILARES, EM RINS DE ASININOS}

(EQUUS ASINUS)

\author{
WILSON MACHADO DE SOUZA \\ Professor Assistente Doutor \\ Faculdade de Ciências Agrárias e \\ Veterinarias de Jaboticabal - UNESP \\ JOAO GILBERTO LOPES PEREIRA \\ Professor Adjunto \\ Faculdade de Medicina Veterinaria e \\ Zootecnia da USP
}

SOUZA, W.M. \& PEREIRA, J.G.L. Contribuigao ao estudo dos elementos vasculares. arteriais e venosos, hilares, justanilares e extrahilares, em rins de asininos (Equus asinus). Rev. Fac. Med. Vet. Zootec. Univ. 5. Paulo, 24(2):127-140, 1987.

RESUMO: O trabalho baseia-se no exame de 30 conjuntos aorangendo rins, vasos, ureteres e tractos correspondentes da arteria aorta e veia cava caudal, retirados de asininos da raca Péga, adultas, 17 machos e $13 \mathrm{fé-}$ meas, nos quais se estudaram mediante disseccáo, apds terem sido as pecas fixadas em solucáo aquosa de formal a $10,0 y$ durante 48 horas, o numero e locais de entrada e salda dos ramas arteriais e as ralzes venasas. hilares, justanilares e extranilares, em diferentes quadrantes, bem camo o comportamento global desies ramos consideracos isoladamente, visando obter maiores substdios para o melhcr connecimento da anatomia dos equlaeos.

UNITERMOS: Anatomia, eqcldeos; Rim; ArtePias: Veias

\section{INTRODUCAO}

Procurando melhor conhecer a disposicaro dos elementos vasculares componentes dos pediculos renais nos animais domésticos e silvestres, realizamos, nesta oportunidade, estudo destes aspectos em asininos, buscando analisar o número, a disposibăo e as regiäes de entrada e salda, respectivamente, dos ramos arteriais e das ralzes venosas, objetivando o conhecimento das aludidas estruturas nesta especie.

\section{MATERIAL E METODO}

Valemo-nos, na presente pesquisa, de 30 pares de rins, retirados de asininos (Equus asinus) da raça Pêga, 17 machos (M) e 13 fêmeas (F), adultos, procedentes de diversas regiaes da criacăo dos estados de 5ro Paulo e Minas Gerais e abatidos no Matadouro de Equideos Primeat em Braganca Paulista, estado de sao Paulo. Apos a evisceracáo dos animais, obtivemos os orgaros ainda unidos por suas artérias e veias aos tractos respectivamente da aorta e da veia cava caudal. Os conjuntos foram fixados en soluça aquasa de formal a $10,0 \%$ durante 48 horas no minimo. En segulda, mediante disseçao, individualizaram-se os componentes dos pediculos renais.

para a descricaro dos resultados, procuramos demarcar os quadrantes craniodorsal, cranioventral, caudodorsal e caudoventral com o auxilio de duas linhas, uma longitudinal, tracada de pola a polo do rim, representando o diametro craniocaudal, e outra equivalente ao diametro dorsoventral, perpendicular à primeira, interceptando-a exatamente no centro do ureter. Delimitados estes territorios, neles 5 ituamos as regioes hilar, justanilar e extranilar a custa de quatro circunferēncias, representando, a menor delas, o ureter (Fig. 1). Por fim, registramos desenhos esquemáticos que configuram os pares de rins submetidos a um giro de $90^{\circ}$ no sentido medioventral, assim como o numero, os locais de entrada e salda, respectivamente dos ramas arteriais e das ralzes venosas, levando-se en conta năo apenas os diferentes quadrantes, mas tambem as regioes neles assinaladas.

Para o estudo estatistico dos resultados utilizamos a analise de variancia e o teste $T$ de "student". 


\section{RESULTADOS}

Com base nas disseccaes dos 30 pares de rins de asininas, expomos os resultaaos, correspondentes ao numero, disposicao e regiás de entrada e salda, respectivamente, dos ramos arteriais e das ralzes venosas, de acordo com os quadrantes e linhas tracadas, considerando separadamente, as regiaes hilar, justahilar e extrahilar couad. $1,2,3,4,5,6)$

\section{COMENTARIOS}

Ds resultados ora registrados para os asininos (Equus asinus), relativos ao numero e localizacão dos ramos arteriais e das ralzes venosas, nos diferentes quadrantes e regioes delimitadas no hilo rena! oestes animais, permitem uma comparacaro com aqueles assinalados por outros autores que valendo-se de metodologia semelhante a que adotamos, utilizaram tambem os equideos em suas pesqui5 as.

Deste modo, PEREIRA, 27, examinando este aspecto em cavalos sem raca definida, verificou que a artéria renal fornece de 3 a 10 ramos, com maior frequôncia oe $5(26,7 \%)$ a direita, e de 3 a 11 ramos, com maior ocorrencia de 5 $(40,0 \%)$ à esquerda para a região hilari de 1 a 6 ramos com maior frequencia de 2 $(26,7 \%)$ a direita, e igualmente de 1 a $E$ ramos, sendo mais frequentes 2 e 4 $(26,7 \%)$ para a regiao justahilari de 1 a 15 com maior ocorrència de $5(16,7 \%)$ a direita e de 1 a 18 ramos com maior frequencia de 3,5 e 6 ( $: 3,3 \%$; a esquerda para a regizo extrahilar.

Por sua vez, AlbuQUERQUe, !, estudando rins de muares, observou que a arteria renal oferece de 2 a 7 ramos com major ocorréncia de $6(30,0 \%)$ à direita, e de 2 a 3 ramos com maior frequencia de 4 a $6(30,0 \%)$ à esquerda para a regizo hiliar; de 1 a 5 ramos, com maior ocorrência de $1(30,0 \%)$ à direita, e de 1 a 7 ramos sendo mais frequente 2 e 3 $(26,7 \%)$ à esquerda para a regiao justahilar; de $i$ a 8 ramos com maior frequencia de 3,5 e $7(16,7 \%)$ à direita, e de 1 a 11 ramos sendo de maior ocorrência de $4(20,0 \%)$ à esquerda para a regiao extrahilar.

De outra parte, GUARENTI, 14, pesquisando o numero e situacro dos vasos renais em cavalos da raca Crioula, evidenciou oue a arteria renal cede de 2 a 10 ramos, sendo inais frequentes 5 $(36,7 \%)$ à direita, e de 1 a 9 ramos com major ocorréncia de $6(33,3 \%)$ a esquerda para a regiao hilar; de 1 a 5 ramos sendo de maior ocorrencia $3(30,0 \%)$ a direita, e de 1 a 7 ramos sendo mais frequente 3 e $5(20,0 \%)$ a esquerda para a regiao justahilar; de 1 a 8 ramos $=0 m$ maior freqúencia de $5(33,3 \%)$ a direita, e de 1 a 7 ramos com maior ocorréncia de $6(23,7 \%)$ a esquerda para a regiao extrahilar.

Estes resultados, se tomados genericamente, destoam daqueles que ora assinalamos para os asininos, quando pudemos constatar que a arteria renat oferece de 1 a 5 ramos com major freqôencia de $2(40,0 \%)$ á direita e, igualmente de 1 a 5 ramos, sendo, de maior ocorrência $3(43,3 \%)$ a esquerda para a regia hilar; de 1 a 5 ramos sendo mais frequentes $3(33,3 \%)$ a direita, e de $q$ a 8 ramos com maior ocorrência de 2 $(30,0 \%)$ a esquerda para a regiao justahilar; de 1 a 6 ramos com maior freqổncia de $1(36,7 \%)$ à direita, e de 1 a 7 ramos com maior ocorrencia de 2 $(20,0 \%)$ a esquerda para a regiao extranilar.

No atinente à veia renal que PEREI RA, 27 consioerou como unica e situada dorsalmente a ureter nos cavalos sem raca definida; ao numero de ralzes venosas observadas por ALBUDUERQUE, 1 , nos muares em numero de uma $(100,0 \%)$ em ambos os rins na regiao hilar, uma $(6,7 \%)$ à direita e uma $(13,3 \%)$ à esquerda na regizo justahilar e, uma $(3,3 \%)$ a dineita e uma $(13,3 \%)$ à esquerda na regiao extrahilar; que GUARENTI, 14. registrou nos cavalos da raca Crioula em numero de uma $(93,3 \%)$ e duas $(5,7 \%)$ à direita e uma $(86,7 \%)$ e duas $(13,3 \%)$ a esquerda na regiao hilar, e duas $(3,3 \%)$ à direita e uma $(15,7 \%)$ à esquerda na regiăo justahilar, estes dados năo se af astam daqueies que observamos em relacaro aos asininos, vale dizer, uma $(83,3 \%)$ e duas $(16,7 \%)$ à direita e uma $(90,0 \%)$ e duas $(10,0 \%)$ à esquerda na regizo hilar, uma $(20,0 \%)$ e duas $(3,3 \%)$ a direita e uma $(20,0 \%)$ a esquerda na regizo justanilar, embora năo tennamos visto, como aLBUQUERQUE, 1 , observou em muares, estes vasos na regiăo extranilar.

Relativamente a localizaço dos ramos arteriais, os vasos encontrados na regizo hilar ocupam, com maior freacencia, tanto à direita como à esquerda nos cavalos sem raca definida, disposicăo prevalentemente periferica, enquanto nos 
Contribuiçăo ao estudo dos elementos vasculares, arteriais e venosos, hilares, justahilares e extrahilares, em rins de a sininos (Equus asinus)

animais da raca Crioula localizam-se em situacaro exclusivamente periférica a direita e predominantemente periférica a esquerda; na regiaro extrahilar, situamse com maior frequência, tanto à direita quanto a esquerda em maior numero no centro da regizo para os cavalos sem raca definida, ao passo que para os animais da raca Crioula, a direita predominam na periferia e a esquerda distribuem-se em igual numero no centro e na periferia. Estes resultados diferem daqueles que ora observamos em relacao aos asininos, onde verificamos tanto para a regia hilar como para a extrahilar, à direita e à esquerda, os vasos arteriais ocupando com maior frequencia - centro das regiŏes. Conforme esclarece AlaUdUerQue, 1, para muares, nao foi posslvel o estudo do comportamento global desses vasos, o que nos impediu a realizaço de qualquer tipo de cotejo. Fato similar ocorreu com refêrencia aos vasos de situacăo justahilar, identificados por PEREIRA, 27 e GUARENTI, 14, pois neste caso entendemos que, por se tratar de regiăo de transiço, torna-se difleil caracterizar as diferentes posiGaes dos vasos.

Na atinente ao povaamento dos quadrantes pelos ramos arteriais, observando os quadros dos trabalhos de PEREIRA, 27. ALBUQUERQUE, 1 e GURRENTI, 14, nos especimes por eles estudados, vimos que - quadrante mais densamente povado tanto a direita como a esquerda, considerando canjuntamente as regiaes hilar. justahilar e extrahilar e o cranioventral, fato coincidente com os dados por nos estabelecidos para os asininos enquanto que, relativamente ao quadrante menos povoado, nossos achados assemelham-se apenas aos registrados para muares, ou seja, um menor numero de ramos arteriais, tanto a direita como à esquerda, para o quadrante caudoventral. Analisando a disposicăo das ralzes venosas, vemos que, tanto para os muares como para os equinos da Raca Crioula, estes vasos situam-se mais freqoentemente sobre a linha dorsoventral, entre os quadrantes craniodorsal e caudodorsal, isto e, 96,7\% a direita e 93,3\% á esquerda para o primeiro caso, e $33,3 \%$ a direita e $86,7 \%$ a esquerda para $0^{\circ}$ segundo caso, dados estes que se aproximam Jaqueles ora consignados em relacao aos asininos, vale dizer, 93,3\% à direita e 96,78 a esquerda.

Je outra parte, PEREIRA, 27, ALBUQUERQUE, 1 e GUARENTI, 14, evidenciaram, bilateralmente, igual número de ramos arteriais em 20,0\%, 13,3\% e 25,7\% e, de raizes venosas em $100,0 \% 83,3 \%$ e $70,0 \%$ respectivamente, resultados estes que se assemelham aos assinalados para os asininos, apenas no que tange aos ramos arteriais, ou seja, 16,7\%.

Os dados caltidas nos compendios de Anatomia Veterinaria permitem-nos apenas algumas comparaças de carater geral. pois seus autares relatam informaçes incompletas e mesmo discordantes sobre o assunto.

CARADONNA, 7, FRUILLI, 11 e 8055I, 2, descrevem a existência de 2 ou 3 ramos oriundos da arteria renal, o que corresponde a 6,7\% dos nossos achados e somente à direita; quanto à referêneia de MANNU, 20, isto e, 4 a 6 ramos, is to equivale a 43,3\% dos nossos dados a direita e 46,7\% à esquerda. GURLT's, 15, ELLENEERGER \& BAUM, 10 , BRUNI \& ZIMMERL 6, SISSON \& GROSSMAN, 30 e SCHWRRZE \& SCHRODER, 29 citam de 5 a 8 ramos, dados estes que correspondem a 63,3\% dos nassos achadas à direita e à esquerda. BRADLEY, 5 , por sua vez, registra 6 a 8 ramos, valor coincidente em 50,0\% das nossas preparacőes à direita e 53,3\% à esquerda. De outra parte, as citacóes de FRANCK, 12, MONGIARDINO, 24, MARTIN, 21, 22, MONTANNE \& BOURDELLE, 25, LEPOUTRE, 18, LESBRE, 19, SCHMALTZ, 28, BOURDELLE \& BRESSOU, 3,4, ZIMMERL, 32, NICKEL et alii, 26, DOBBERSTEIN \& HOFFMAN, 8,9, KOCH, 16, 17, ZANOLLI, 31, MASSUI, 23 e GONZRLEZ Y GRRCIA \& GONZALEZ ALUAREZ, 13. năo nos permitem qualquer tipo de cotejo. Embora GURLT'S, 15, FRANCK, 12, MARTIN, 21, 22, BRADLEY, 5, LESBRE, 19, SCHMALTZ, 28, ELLENBERGER \& BRUM, 10, SISSON \& GROSSMAN, 30 e SCHWARZE \& SCHRODER, 29 esclarecam que os ramos arteriais ganham o rim pela face ventrab, observamos estes vasos adentrando - drgaro tamberm pela sua face dorsál.

Finalmente, no tocante as ralzes venosas, nossos achados de uma $(63,3 \%)$, duas $(30,0 \%)$ e très $(6,7 \%)$ a direita e uma $(70,0 \%)$ ou duas $(30,0 \%)$ a esquerda correspondem parcialmente as informaças de BRUNI \& ZIMMERL, 6 , DCBERSTEIN \& HOFFMANN, 8,9, ELLENBERGER \& BAUM, 10, FAUILLI, 11. LEPOUTRE, 18, LESBRE, 19, MANNU, 20, MARTIN, 21, 22, MASSUI, 23, NICKEL et alii, 25, SCHWARZE \& SCHRODER, 29 e ZIMMERL, 32 que relatam a presenca de uma unica raiz venosa e afastam-se dos de BOSSI, 2 , e CARAOONiNA, 7 que consideram, respectivamente, 4 ou 5 e 5 ou 6 ralzes constitulndo aquele vaso.

\section{CONCLUSOES}

Ds resultados ora ootidos em 30 
pares de rins reijrados de asininos (Equus asinus), da raca Pêga, adultos, 17 machos e 13 fêmeas, relativos ao ndmero e locais de entrada e salda dos ramos arteriais e das ralzes venosas, hilares, justahilares e extranilares, nos diferentes quadrantes, levam-nos às seguintes conclusores:

1. Os ramos arteriais situados na regia hilar săo identificados no rim direito em numero de dois $(40,0 \%)$ trés $(36,7 \%)$, quatro $(10,0 \%)$, einco $(5,7 \%)$, um $(5,7 \%)$ e, no esquerdo, em numero de três $(43,3 \%)$ dois $(36,7 \%)$, quatro $(10,0 \%)$, um $(6,7 \%)$, cinco $(3,3 \%)$.

2. Ds ramos arteriais contidos na regia justahilar săo registrados no rim direito em numero de trés $(33,3 \%)$, um $(26,7 \%)$, einco $(16,7 \%)$, dois $(13,3 \%)$, quatro $(6,7 \%) e$, no esquerdo, em número de dois $(30,0 \%)$, três $(23,3 \%)$, quatro $(13,3 \%)$, um $(13,3 \%)$, cinco $(10,0 \%)$, seis $(3,3 \%)$, oito $(3,3 \%)$.

3. Ds ramos arteriais computados na regiă extrahilar săo assinalados, no rim direito, em numero de um $(36,7 \%)$, dois $(13,3 \%)$, quatro $(10,0 \%)$, três $(6,7 \%)$, cinco $(3,3 \%)$, seis $(3,3 \%)$, e, no esquerdo, em número de dois $(20,0 \%)$, um $(13,3 \%)$, très $(13,3 \%)$, quatro $(10,0 \%)$, einco $(3,3 \%)$, sete $(3,3 \%)$.

4. No rim direito, como no esquerdo, o quadrante cranioventral e o mais intensamente povado pelos ramos arteriais, seguido dos quadrantes craniodorsal, caudodorsal e caudoventral.

5. Os vasos arteriais ocupam posicăo freqoentemente central 19 vezes $(63,3 \%)$ a direita e 12 vezes $(40,0 \%)$ à esquerda; distribuem-se em igual numero tanto para os quadrantes ventrais como para os dorsais, 5 vezes $(16,7 \%)$ à direita e 7 vezes $(23,3 \%)$ à esquerda; encontram-se em menor numero nos quadrantes ventrais 6 vezes $(20,0 \%)$ a direita e 11 vezes $(36,7 \%)$ a esquerda e concentram-se mais nos quadrantes craniais 30 vezes $(100,0 \%)$ a direita e 29 vezes $(96,7 \%)$ à esquerda.
5. Na regia hilar, os ramos arteriais saro unicamente centrais 12 vezes $(40,0 \%)$ a direita e 15 vezes $(50,0 \%)$ a esouerda, em igual numero tanto no centro como na periferia da regiao 7 vezes $(23,3 \%)$ a direjta e 5 vezes $(16,7 \%)$ a esquerda; predominantemente perifericos 6 vezes $(20,0 \%)$ à direita e 4 vezes $(13,3 \%)$ à esquerda; em maior numero no centro da regiao 5 vezes $(16,7 \%)$ à direita e 5 vezes $(16,7 \%)$ à esquerdai somente perifericos, 1 vez $(3,3 \%)$ à esquerda.

7. Na regiăo extrahilar, os ramos arteriais sao unicamente centrais 11 vezes $(36,7 \%)$ a direita. e 13 vezes $(43,3 \%)$ à esquerda, preponderantemente centrais 5 vezes $(16,7 \%)$ a direita e 3 vezes $(10,0 \%)$ a esquerda; igualmente centrais a perifericos, 3 vezes $(10,0 \%)$ à direita e 1 vez $(3,3 \%)$ à esquerda; ape nas perifericos 2 vezes $(6,7 \%)$ à direita; em maior numero parifericos, 2 vezes $(6,7 \%)$ à esquer. da.

8. As ralzes venosas situam-se sobre a linha dorsoventral, entre os quadrantes craniodorsal e caudodorsal, 28 vezes $(93,3 \%)$ a direita e 29 vezes $(96,7 \%)$ à esquerda; no quadrante caudodorsal, 10 vezes $(33,3 \%)$ à direita e 5 vezes $(16,7 \%)$ a esquerda; no quadrante craniodorsal 5 vezes $(16,7 \%)$ a direita e 2 vezes $(G, 7 \%)$ a esquerda, no quadrante caudoventral 2 vezes $(6,7 \%)$ apenas à esquerda.

9. As ralzes venosas sao contadas, na regiao hilar, em número de uma $(83,3 \%)$ e duas $(16,7 \%)$ à direita e uma $(90,0 \%)$ e duas $(10,0 \%)$ à esquerda, enquanto na regiao justahilar uma $(20,0 \%)$ e duas $(3,3 \%)$ à direita e uma $(20,0 \%)$ à esquerda e ocupam, na regiao hilar, posiczo predominantemente centrai.

10. Nos 30 pares de orgaros examinados, para os rins direito e esquerdo, correspondentes, encontramos igual numero de ramos arteriais apenas 5 vezes $(16,7 \%)$ e de ralzes venosas 15 vezes $(50,0 \%)$.

11. Quanto ao comportamento global dos ramas arteriais, estes localizam-se em igual numero, tanto a direita como à esquerda, na regiăo hilar $g$ 
Contribuiçāo ao estudo dos elementos vasculares, arteriais e venosos, hilares, justahilares e extrahilares, em rins de a sininos (Equus asinus)

vezes $(30,0 \%)$, vale dizer 6 vezes $(20,0 \%)$ unicamente no centro; 2 vezes $(6,7 \%)$ em igual ndmero no centro e na periferia da regia e 1 vez (3,3\%) em maior numero no centro da regiao; e, na regiăo extrahilar 10 vezes $(33,3 \%)$, isto e, 7 vezes $(23,3 \%)$ somente no centro, 2 vezes $(6,7 \%)$ prevalentemente no centro e apenas 1 vez $(3,3 \%)$ em ndmero equivalente tanto no centro como na periferia da regia.

12. O estudo estat1stico dos resultados nao demonstrou existir diferencas significantes, ao nlvel de 5,0\%, quanto ao sexo, no que tange ao numero de ramos arteriais e de ralzes venosas.

13. Confiontando, isoladamente, o numero de ramos arteriais e ralzes venosas dos asininos, observados em cada regiăo estudada, com os encontrados na literatura relativa aos cavalos sem raca definida, Erioulas e Muares, verificamas existir diferencas estatisticamente significantes, ao nivel de $5,0 \%$ apenas no aue tange aos ramos arteriais das regiăes hilar e extrahilar, em tados os casos, tanto para o rim direito como para o esquerdo.

SOUZR, W.M. \& PEREIRA, J.G.L. Contributian to the study of the vascular arterial and venous elements - hilar, justahilar and extrahilar - in kidneys of asinine. Rev. Fac. Med. Vet. Zootec. Univ. 5. Paulo, 24(2):127-140, 1987.

\begin{abstract}
SUMMRRY: This work is based on the examination of 30 adult animals of the Pega race - 17 male and 13 female - whose kidneys, vessels, ureters and tracts related to the aorta artery and caudal vena cava dere extracted and studied. This was done by dissection after the oieces were fixed in aqueous solution of formol at $10.0 \%$ for 48 hours. The number and sites of penetration and outlet the arteriat branches and the hilar, justahilar and extrahilar venous roots was verified in the different quadrants, as well as the glooal behaviour os these branches.
\end{abstract}

UNITERMS: Gnatomy of horses; Kioney; Arteries; Veins

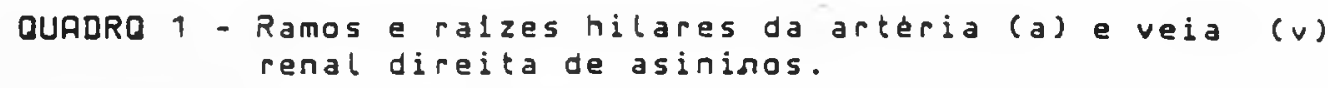

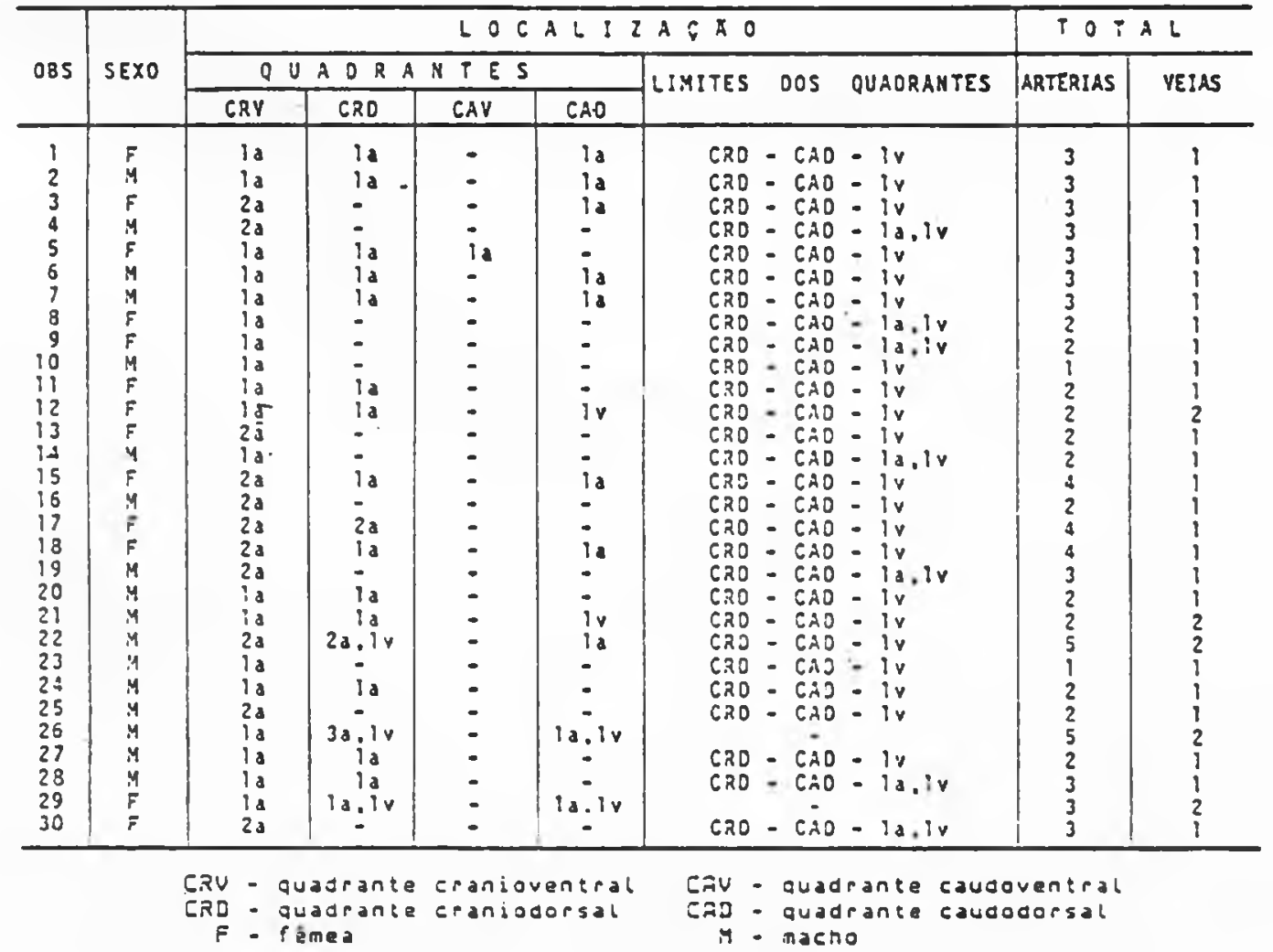


132 SOUZA, W.M. \& PEREIRA, J.G.L.

QUADRO 2 - Ramos e ralzes justahilares da arteria (a) e veia (v) renal cireita de asininos.

\begin{tabular}{|c|c|c|c|c|c|c|c|c|c|c|}
\hline \multirow{3}{*}{035} & \multirow{3}{*}{ SEXo } & \multicolumn{7}{|c|}{$\angle O C A L I Z A G Z O$} & \multicolumn{2}{|c|}{$T O Y A L$} \\
\hline & & \multicolumn{4}{|c|}{ QUA $2 R A N T E S$} & \multirow{2}{*}{ LIMITES } & \multirow{2}{*}{ DOS } & \multirow{2}{*}{ Q'JADRANTES } & \multirow{2}{*}{ ARTERIAS } & \multirow{2}{*}{ VEIAS } \\
\hline & & CRV & CRO & CAV & CAD & & & & & \\
\hline 1 & $F$ & do & - & id & • & & - & & 2 & - \\
\hline 2 & $\dot{M}$ & id & 28. & - & - & & - & & 3 & $=$ \\
\hline $\begin{array}{l}3 \\
4\end{array}$ & M & 28 & 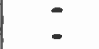 & $\because$ & ${ }^{2 a} \mathrm{ia}^{\mathrm{s}}$ & & : & & $\begin{array}{l}3 \\
3\end{array}$ & $!$ \\
\hline 5 & $\boldsymbol{F}$ & $i_{a}$ & 28 & 22 & $\because$ & & - & & 5 & - \\
\hline 6 & $M$ & - & $1 \mathrm{a}$ & 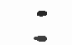 & 38 & & $=$ & & 1 & i \\
\hline 8 & $\vec{F}$ & la & - & - & $12: 18$ & & $\overline{-}$ & & 1 & ! \\
\hline 10 & F & $l_{a}$ & 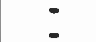 & $\therefore$ & : & & $\overline{-}$ & & i & - \\
\hline$i$ & $\vec{F}$ & 28 & la & ia & $12,1 \mathrm{v}$ & & 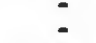 & & $\begin{array}{l}1 \\
5\end{array}$ & i \\
\hline 12 & F & 12 & 1 & - & 3 & & - & & 5 & - \\
\hline $\begin{array}{l}13 \\
14 .\end{array}$ & M & $\frac{2 a}{4 a}$ & $3 a, I_{y}$ & - & $i_{\mathrm{a}}$ & & 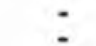 & & $\begin{array}{l}3 \\
5\end{array}$ & i \\
\hline 15 & $F$ & $:$ & $i_{a}$ & - & - & & - & & 1 & : \\
\hline 16 & $\underset{F}{M}$ & $l_{a}$ & - & $1 \mathrm{a}$ & : & & - & & 2 & - \\
\hline 18 & F & - & 1. & $=$ & ia & CRV & - CRD & $-1 a$ & 3 & $:$ \\
\hline 19 & $M$ & - & - & - & $2 a$ & CAV & - CAD & -10 & 3 & - \\
\hline 20 & $M$ & $2 a$ & $:$ & $:$ & $i_{\mathrm{a}}$ & CAV & - CAD & $-1 \mathbf{a}$ & 1 & $:$ \\
\hline 22 & M & $2 a$ & ; & : & ia,iv & & - & & 3 & $i$ \\
\hline $\begin{array}{l}23 \\
24\end{array}$ & $M$ & $2 a$ & la & $2 a$ & $:$ & & $\overline{-}$ & & 5 & - \\
\hline 25 & $M$ & ia & $2 a$ & 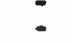 & $i_{\mathrm{a}}$ & & 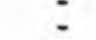 & & $\frac{2}{4}$ & $=$ \\
\hline 26 & $M$ & - & $\because$ & - & - & & - & & $=$ & - \\
\hline 27 & $\mu$ & $2 a$ & $1 a, 1 v$ & - & $1 \mathrm{a}, 1 \mathrm{v}$ & & - & & 2 & 2 \\
\hline $\begin{array}{l}28 \\
29\end{array}$ & $\mathrm{~F}$ & ia & 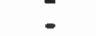 & - & : & CAV & - CAD & $-1 \mathrm{a}$ & 3 & : \\
\hline 30 & $\mathbf{F}$ & 12 & 12 & - & $22,1 v$ & & . & & 4 & $i$ \\
\hline
\end{tabular}

QUADRO 3 - Ramos e raizes extrahilares da arteria (a) e veia (v) renal direita de asininos.

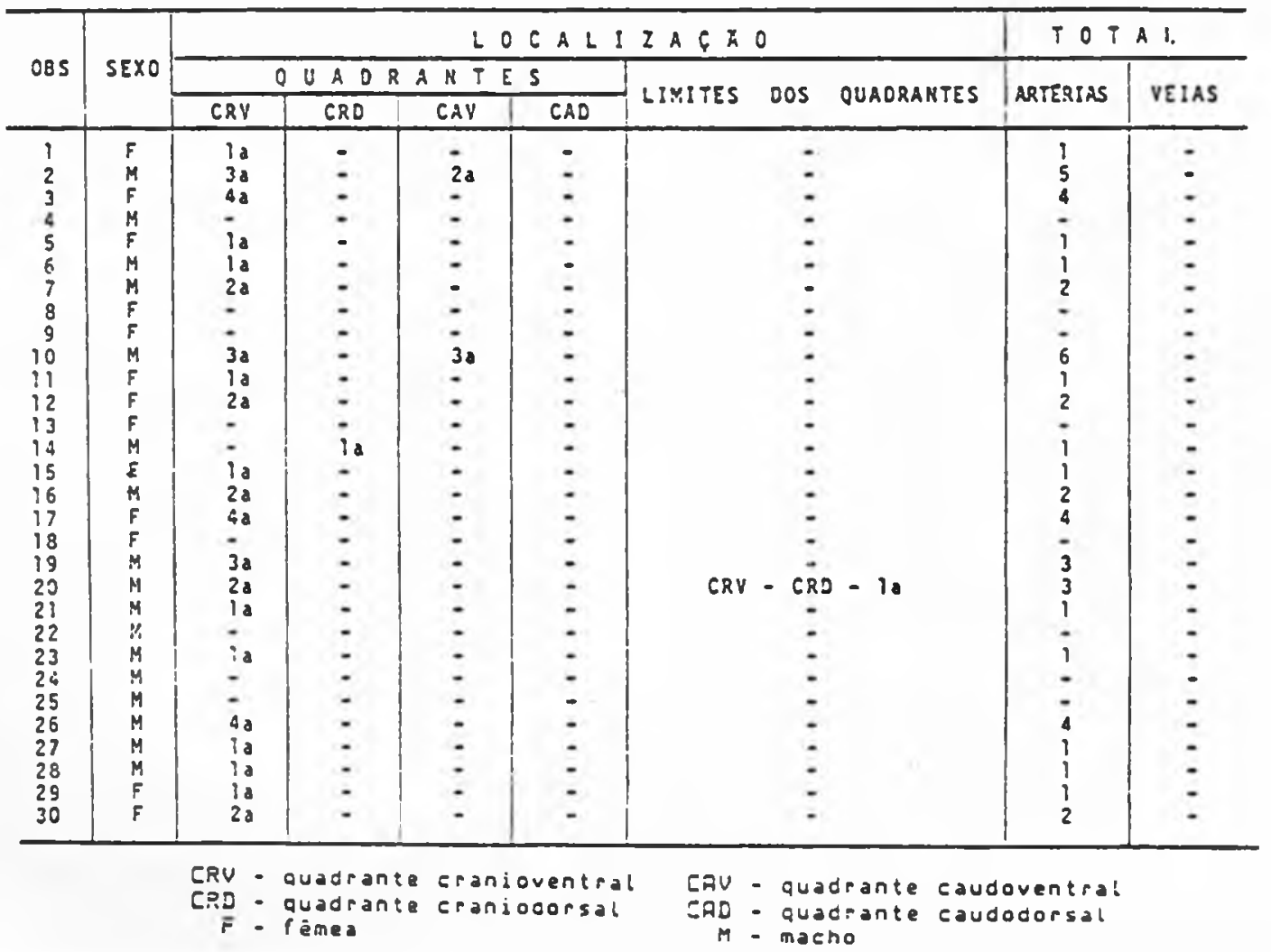


Contribuiçāo ao estudo dos elementos vasculares, arteriais e venosos, hilares, justahilares e extrahilares, em rins de asininos (Equus asinus) QUADRO 4 - Ramos e ralzes da arteria (a) e veia (v) renal
esquerda de asininos.

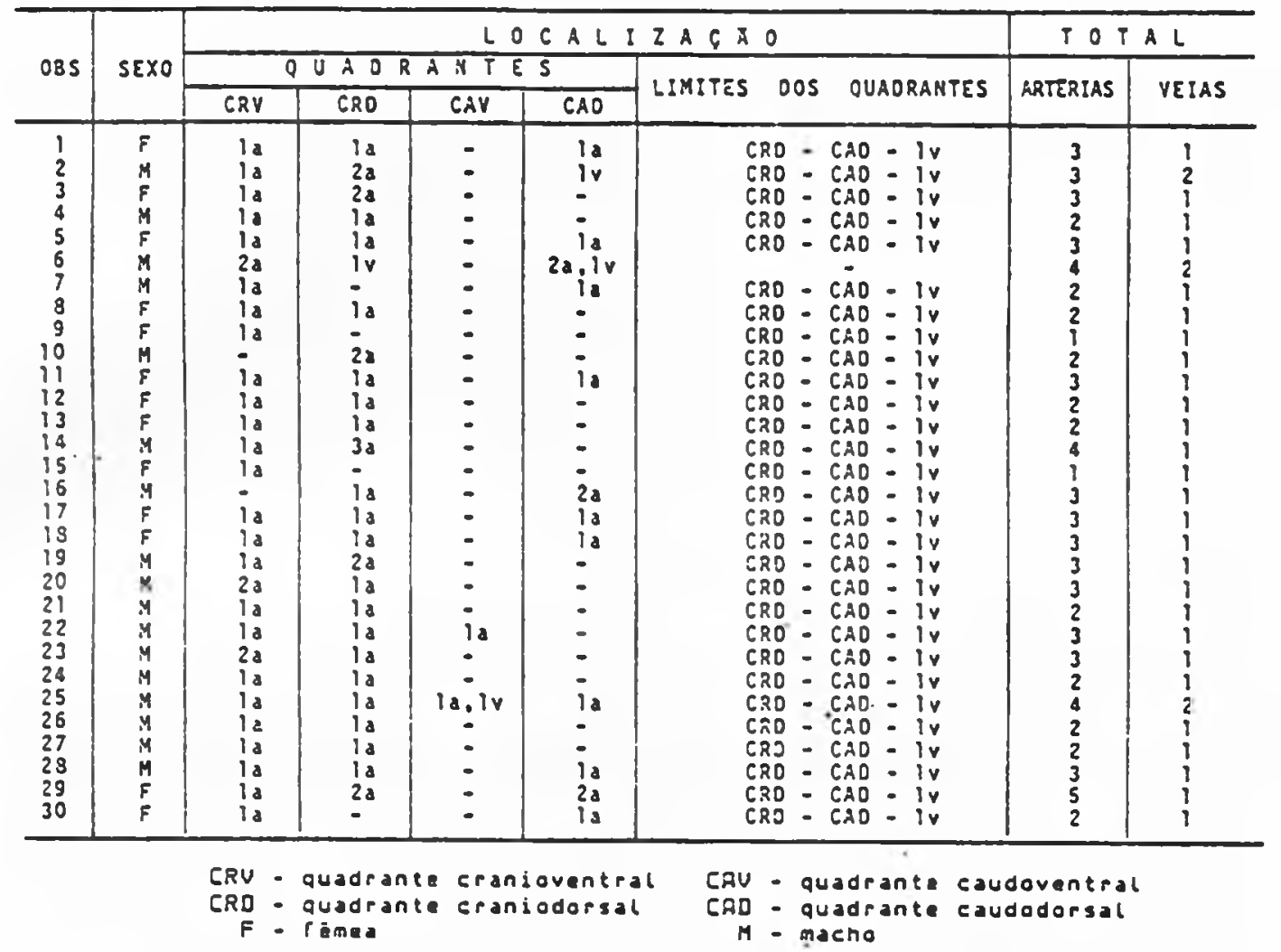

QURDRO 5 - Ramos e ralzes justahilares da artéia (a) e veia $(v)$ renal esquerda de asininos.

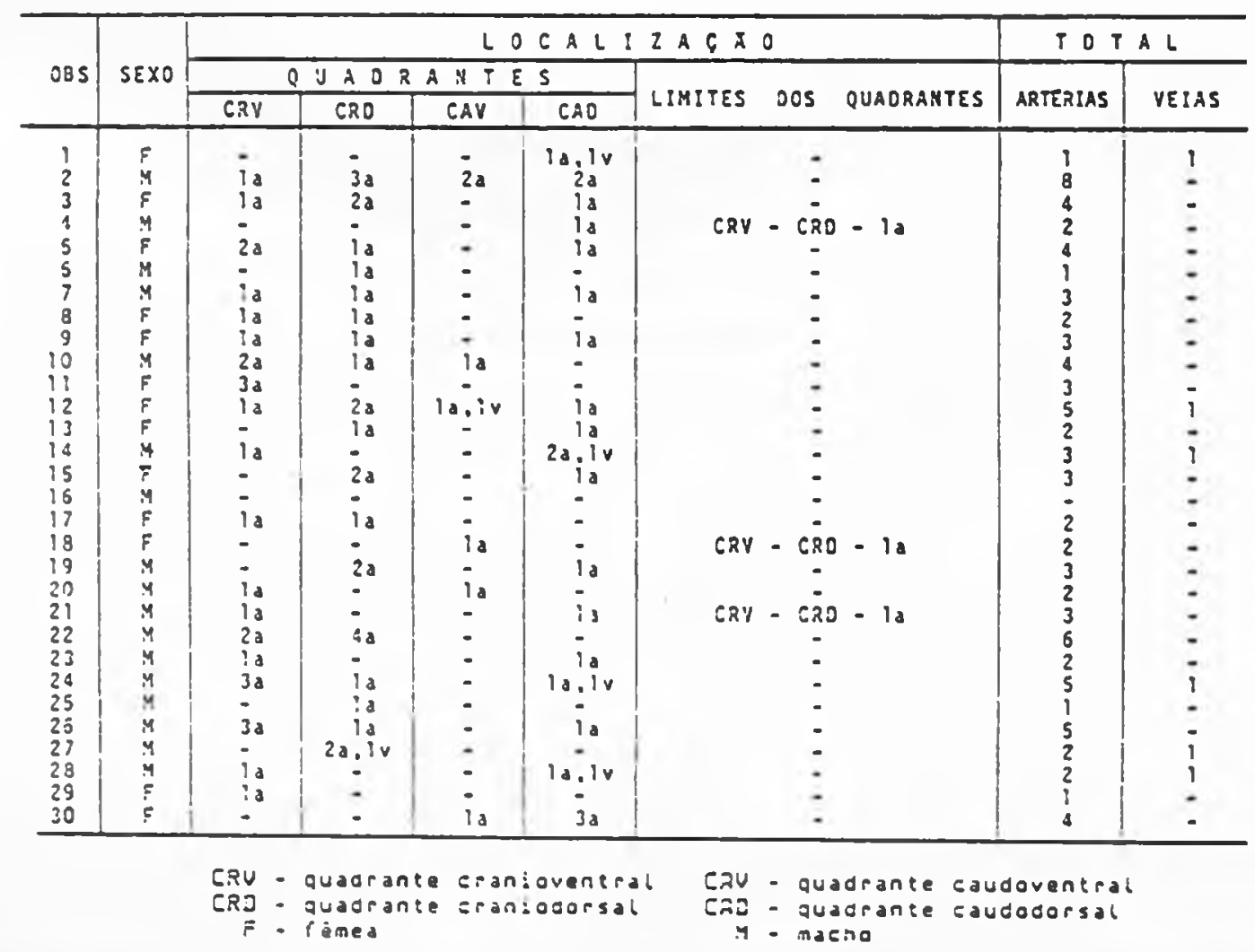


134 SOUZA, W.M. \& PEREIRA, J.G.L.

OUADRo 6 - Ramos e ralzes extrahilares da arteria (a) e veia ( $v$ ) renal esquerda de asininos.

\begin{tabular}{|c|c|c|c|c|c|c|c|c|c|c|}
\hline \multirow{3}{*}{ OBS } & \multirow{3}{*}{ SEXo } & \multicolumn{7}{|c|}{$\angle O C A L I Z A C A O$} & \multicolumn{2}{|c|}{ TOTAL } \\
\hline & & \multicolumn{4}{|c|}{ OUA ORANTES } & \multirow{2}{*}{ LIMITES } & \multirow{2}{*}{ DOS } & \multirow{2}{*}{ QUADRANTES } & \multirow{2}{*}{ ARTERIAS } & \multirow{2}{*}{ VEIAS } \\
\hline & & CRV & CRO & CAV & CAD & & & & & \\
\hline 1 & $\boldsymbol{F}$ & 20 & - & - & - & & - & & 2 & \\
\hline 2 & M & $6 a$ & Is & - & - & & - & & 7 & - \\
\hline 3 & F & ¿a & - & - & - & & - & & 4 & - \\
\hline 8 & $g_{g}^{M}$ & $:$ & $:$ & $:$ & $\overline{-}$ & & $=$ & & : & $=$ \\
\hline $\begin{array}{l}5 \\
6\end{array}$ & M & ia & 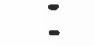 & $:$ & - & & - & & i & $\xi$ \\
\hline 7 & M & - & - & - & - & & - & & - & - \\
\hline 8 & 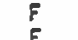 & $:$ & $\overline{-}$ & 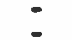 & $=$ & & $z$ & & : & - \\
\hline 10 & M & 3 a & - & - & $=$ & & $=$ & & j & $=$ \\
\hline 11 & F & 30 & 18 & - & - & & - & & 4 & - \\
\hline 12 & F & is & $:$ & $\overline{-}$ & $\overline{-}$ & Cay & - cór & -1 & ; & - \\
\hline 14 & M & 3a & - & - & - & Cot & - & & 3 & $=$ \\
\hline 15 & F & is & is & - & - & & - & & : & - \\
\hline 16 & y & la & la & - & - & & - & • & 2 & 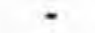 \\
\hline 17 & $\frac{F}{F}$ & $2 a$ & - & $\overline{-}$ & $\overline{-}$ & & $E$ & & $?$ & $\because$ \\
\hline 19 & $M$ & $3 a$ & 28 & - & - & & - & & 5 & - \\
\hline 20 & $M$ & $2 \mathrm{a}$ & - & $\overline{-}$ & $\overline{-}$ & & - & & 2 & $\cdot$ \\
\hline 22 & M & 3a & ia &. & . & & - & & 4 & 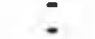 \\
\hline 23 & M & 20 & - & - & - & & - & & 2 & - \\
\hline 24 & M & - & - & $=$ & - & & - & & $=$ & - \\
\hline 25 & M & - & 10 & - & - & & - & & 1 & - \\
\hline $\begin{array}{l}26 \\
27\end{array}$ & 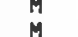 & $\overline{3 a}$ & $\overline{-}$ & $\overline{-}$ & $\overline{-}$ & & - & & i & 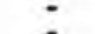 \\
\hline 28 & M & ia & - & - & $=$ & & - & & i & - \\
\hline 29 & $F$ & : & ; & - & - & & $\cdot$ & & & - \\
\hline 30 & $\boldsymbol{F}$ & 28 & la & - & - & & - & & 3 & - \\
\hline
\end{tabular}


Controuiç̧̃o ao estudo dos elementos vasculares, arteriais e venosos, hilares, justairilares e extrahilares, em rins de asininos (Equus asinus)
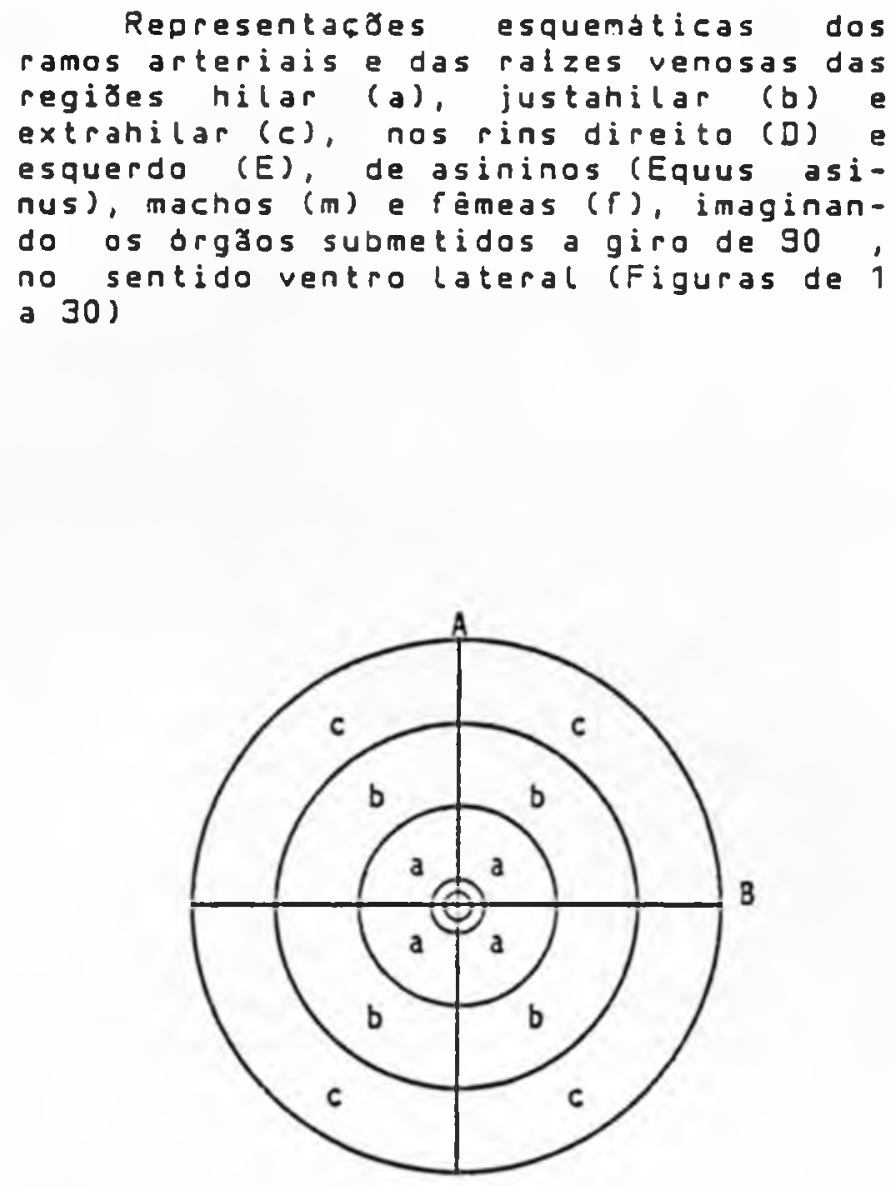

FIGURA 1

A - linha craniocaudal

B - linha dorsoventral

I - quadrante craniodorsal

II - quadrante cranioventral

[1] - quadrante caudodorsal

IV - quadrante caudoventral

(O) - ureter

- ramo arterial

- raiz venosa 
136 SOLZA, W.M. \& PEREIRA. J.G.L.
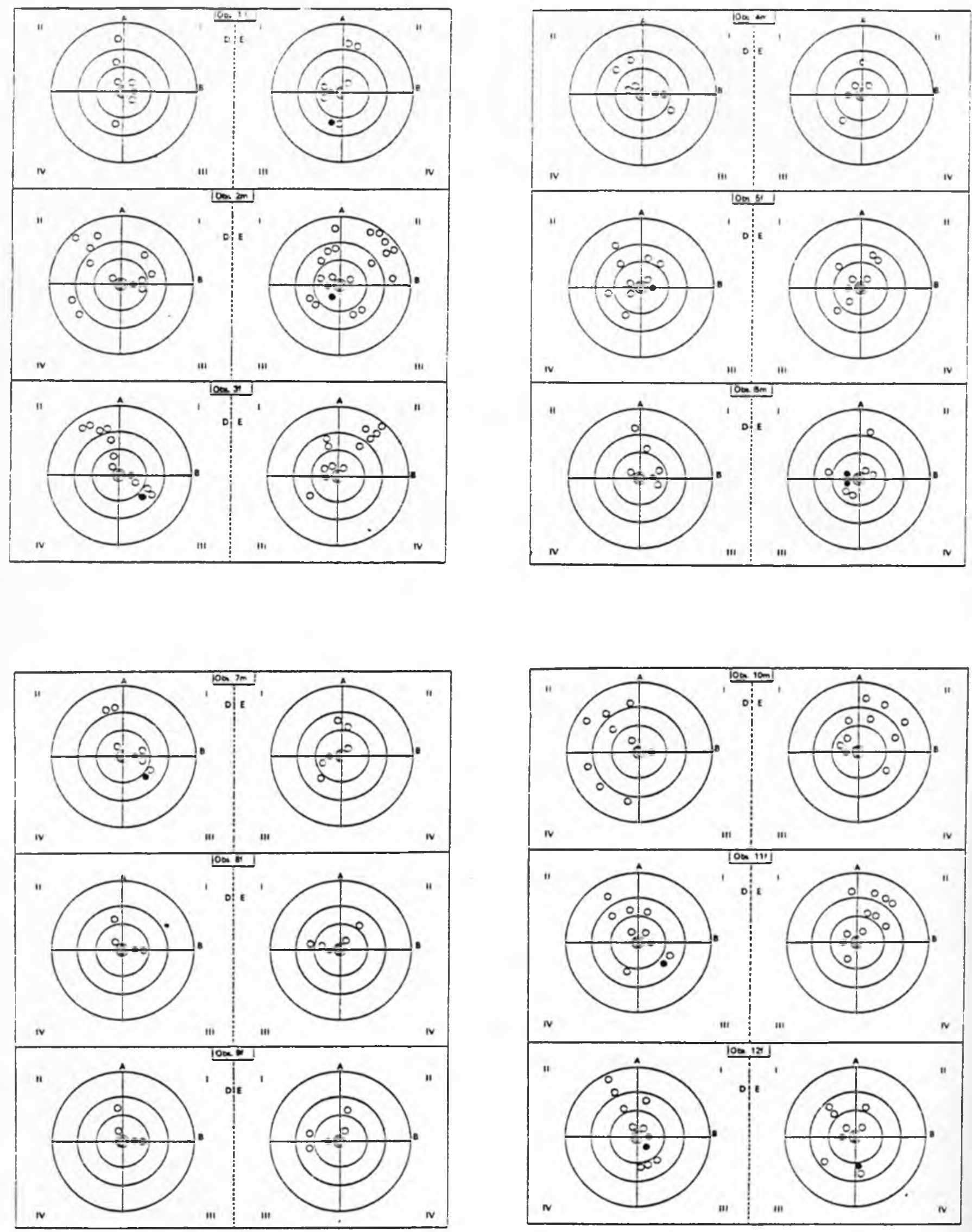

Rev.Fac.Med.Vet.Zootec.Univ.S.Paulo, 24(2):127-140, 1987. 
Contribuiçð̃o ao estudo dos clementos vasculares, arteriais e venosos, hilares, justahilares e extrahilares. em rins de asininos (Equus asinus)
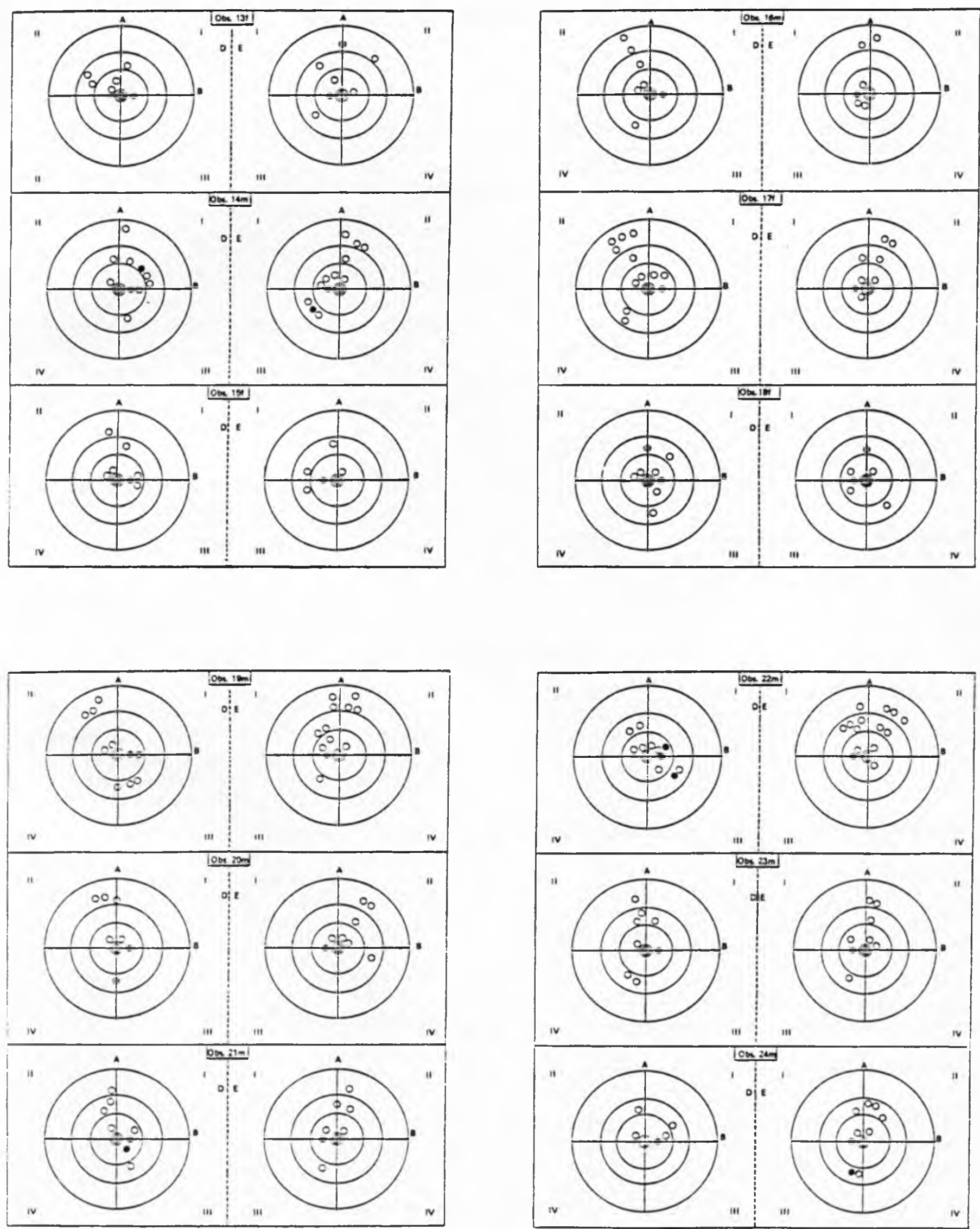

Rev.Fac..Yed.Vet.Zuotec.Univ.S.Paulo, 2+(2):127.140, 1987. 
138 SOUZA, W.M. \& PEREIRA, J.G.L.
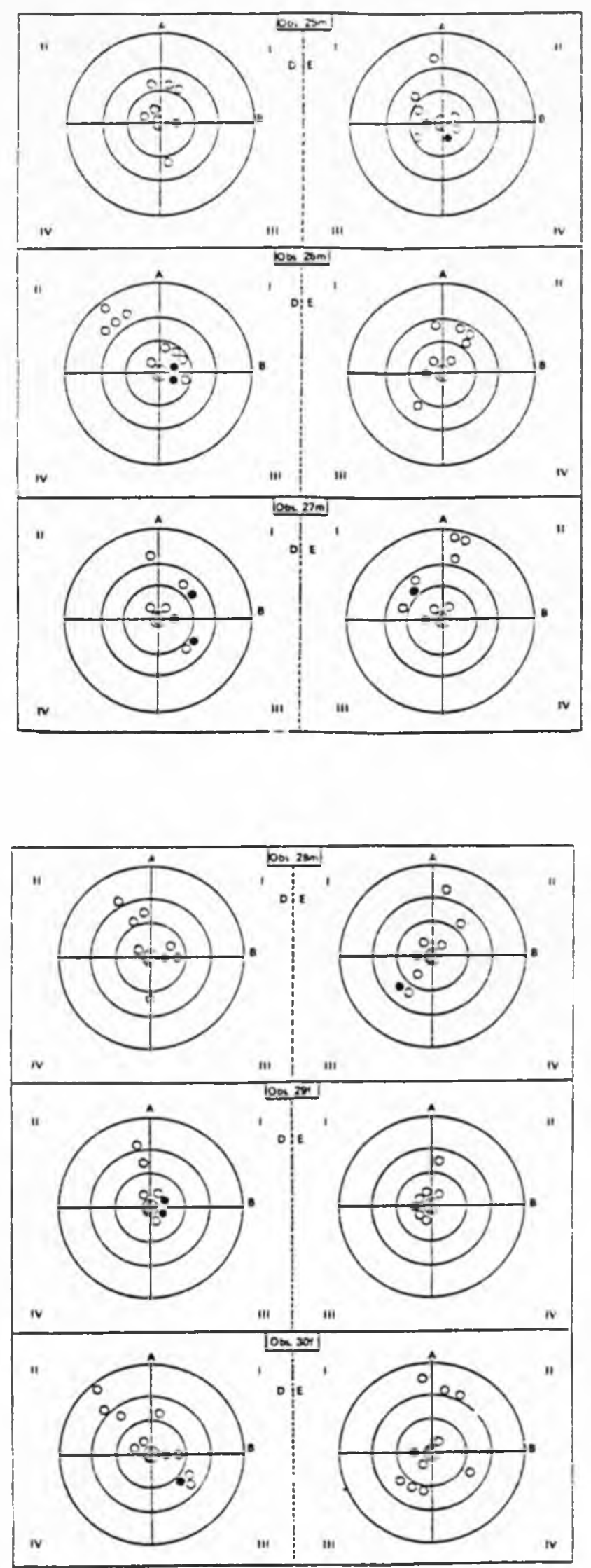

Rev.Fac.Med.Vet.Zootec.Univ.S.Paulo, 24(2):127-140, 1987. 
Contribuiçāo ao estudo dos elementos vasculares, arteriais e venosos, hilares, justahilares e extrahilares,

1 - alguduerque, J.F.g. Contribuifá ao estudo dos ramos arteriais e das ralzes venosas, hilares, justahilares e extrahilares em rins de muares. (Equus caballus $x$ Equus asinus). Saro Paulo, 1976. IDissertacão de mestrado - Escola Paulista de Medicinal

2 - BOSSI, U.; CARADONNA, G.B.; SPAMPANI, G.; UARALDI, L.; ZIMMERL, U. Trattato oi anatomia veterinaria. Milano, Francesco Vallardi, s.d. v.2., p.207, 340, 797.

3 - bourdelle, e. \& Bressou, C. Anatomia régionale des animaux domestiques. Paris, J.B. Bailliere et Fils, 1937. p.72.

4 - bourdelle, e. \& bressou, c. Anatomia régionale des animaux domestiques. paris, J.B. Baillière et Fils, 1938. v.1, D.764-765.

5 - BRRDLEY, O.C. The topographical anatomy of the thorax and abdomen of the horse. Edinburg, w. Green \& Son: 1922. p.147.

6 - BRUNI, A.C. \& ZIMMERL, U. Anatomia degli animali domestici. Milano, Francesco Vallardi, 1947. v.2, $0.193,352$.

7 - CRRADONNA, G.3. Appareschio urogenitale. In: ZIMMERL, $U$. Trattato de anatomia veterinaria. Milano, Francesco Vablardi, 1930.v.3, p.15.

8 - DOBBERSTEIN, Lehrbuch Anatomie Leipzig, o. 136 .

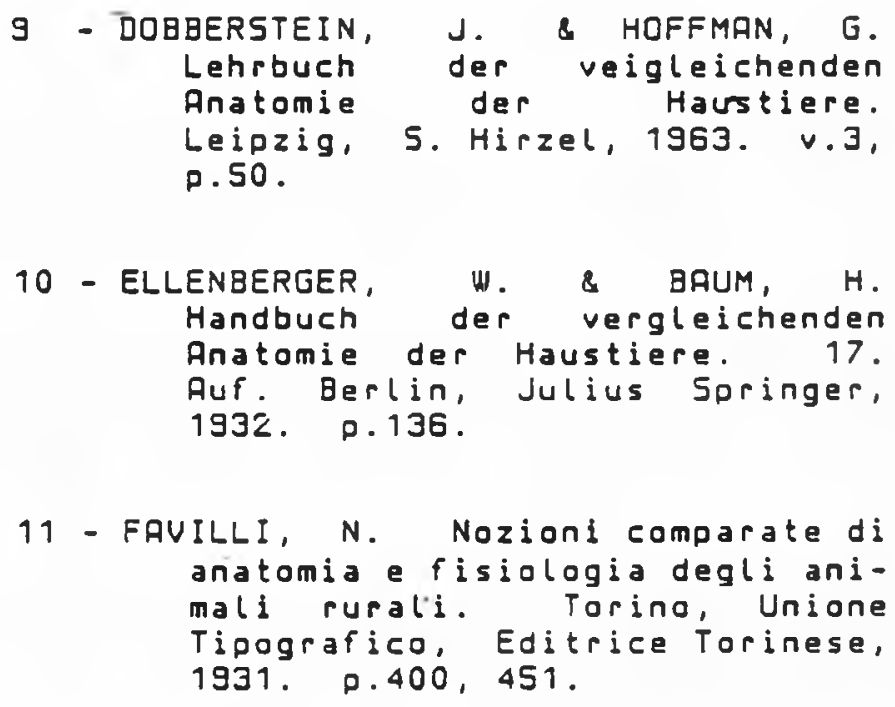

10 - ElLENBERGER, W. \& BRUM, H. Handbuch der vergleichenden Anatomie der Haustiere. 17. Auf. Berlin, Julius Springer, 1932. p. 136 .

11 - FRUILLI, N. Nozioni comparate di anatomia e fisiologia degli animali rurali. Torino, Unione Tipografico, Editrice Torinese, 1931. $0.400,451$.

12 - FRANCK, L. Handbueh der Anatomie der Haustiere. Stuttgart, Schick hardt \& Ebner, 1883. p. 872 .

19 - GONZRLEZ Y GARCIA, J. \& GONZALEZ ALUAREZ, R. Anatomia de los animales domesticos. 7 .ed. Madrid, Grafica Canales, 1961. o.631, 712.

14 - GURRENTI, U.P.J. Contribuigao ao estudo dos ramos arteriais e das ralzes venosas hilares, justahilares e extrahilares, em rins de cavalos da raca Crioula. Pelotas, 1979. / Tese para titular Universidade Federal de Pelotasl

15 - GURLT'5, E.F. Handbuch der vergleichenden Anatomie der Haus-5auge tiere. Berlin, August Hirschwald, 1873. p.620.

16 - KOCH, T. Lehrbuch der VeterinarAnatomie. Jena, Gustav Fischer, 1963. v.2, p. 234.

17 - KOCH, T. Lehrbuch der Veterinar- 
Anatomie. Jena, Gustav Fischer, 1965. v. 3, ว.119.

18 - LEPOUTRE, L. Notes du cours d'anatomie comparee des animaux domestiques. Gemblaux, J. Duculot, 1921. p.154.

19 - LESBRE, F.X. Précis d'anatomie comparee des animaux domestiques. Paris, J.B. Baillière et Fils, 1923. v.2, D.78, 338 .

20 - MANNU, A. Apoarecenio vascolare. In: ZIMMERL, U. Trattato di anatomia veterinaria. Milano, Francesco Vallardi, 1930. v.2, p. 168, 288 .

21 - MARTIN, $P$. Lehrbuch der Anatomie der Haustiere. Stuttgart. Schick-hardt \& Ebner, 1904. p.694, 861.

22 - MARTIN, P. Lehrbuch der Anatomie der Haustiere. Stuttgart, Schick-hardt \& Ebner, 1915. v. 2, p. 172 .

23 - MASSUI, K. Katiku hikaku kaibou gaku. [Anatomia comparada dos animais domesticos] 10.ed. Tokio, Yokendo, 1960. v. 1 . p. 172 .

24 - MONGIARDINO, T. Trattato di anatamia topografica dei mamiferi domestici. Torino, Luigi Delgrosso, 1903. p.192.

25 - mONTANE, L. \& BOURDELLE, E. Anatomie régionale des animaux domestiques. Paris, J.B. Bailliere et Fils, 1913. v.1, p. $862-863$.
26 - NICKEL, R.; SCHUMMER, R.; SEIFER!E, E. Lehrbuch der lnatomie der Haustiere. Berlin, Paul Parey, 1960. v.2, p. 290.

27 - PEREIRA, J.G.L. Contribuigão ao estudo dos ramos arteriais hilares, justahilares e extrahilares em rins de cavalos (Equus cabal(us). Rev. Fac. Med. Vet. Zootec. Univ. 5. Paulo, 11:237$261,1974$.

28 - SCHMALTZ, R. Anatomie des Pieroes. Berlin, Richard Schaeltz, 1928. p.505.

29 - SCHWARZE, E. \& SCHRODER, L. Compendio de anatomia veterinaria. Zaragoza, Acribia, 1972. v.3, p. 72, 98 .

30 - 5ISSON, 5. \& GROSSMAN, J.D. Anatomia de los animales domesticos. Barcelona, Salvat Editores. 1959. p.559.

31 - ZRNOLli, . Manual di Anatomia Veterinaria. La Plata, Felix F.Santi, 1910. v.1, p.982.

32 - ZIMMERL, U. Anatomia Topografica Veterinaria. Milano, Francesco Vallardi, 1949. p.352.

Recebido para publicacăo em 15/04/87 Aprovado para publicacgo em 03/07/87 